\begin{tabular}{|l|l|l|l|}
\hline Eiszeitalter u. Gegenwart & 37 & $\begin{array}{l}31-39 \\
1 \mathrm{Abb} .\end{array}$ & Hannover 1987 \\
\hline
\end{tabular}

\title{
Die Vergletscherung des Belchengebietes (Südschwarzwald) zur Würmeiszeit
}

\author{
GILBERT RAHM *) \\ Upper Pleistocene, Würm glaciation, glacial features, moraines, cirques, glacial morphology \\ Black Forest, Mount Belchen region, Baden-Württemberg \\ TK 25: Nr. $8112,8113,8212,8213$
}

Kurzfassung: Hauptzentren der Vereisung waren neben dem Belchen-Heidstein der Köhlgarten im SW und Breitnauer Kopf im NW. Im Würmmaximum vereinigten sich die Eismassen im E mit dem Wiesetalgletscher. Im W waren die Gletscherzungen infolge der starken Reliefenergie (26-36\%) nur kurz; außerdem sind die Dokumente spärlich. Das besterhaltene Kar ist der Nonnenmattweiher; weitere 5 Kare sind bekannt. Meist aber sind es mehr Kartrichter, die in dem stark reliefierten Gebiet entstanden sind. Von den Rückzugsständen ist der Titiseestand sehr schlecht durch Moränen dokumentiert. Er kann aber, wie die anderen Rückzugsstände auch, durch die Berechnung der Schneegrenze festgestellt werden. Der Falkau-ZipfelhofStand hat an mehreren Stellen deutlichere Moränen hinterlassen; der Waldhofstand ist am besten im Ort Multen belegt. Zur Zeit des Feldsee-Standes war nur noch der Gipfel des Belchen mit Eis bedeckt, und im Nonnenmattweiher hielt sich ein letzter Eisrest. An glazigenen Formen sind weiterhin viele Rundhöcker, Trogtäler und breite Talmulden vorhanden. Die Krinne N des Belchen ist eine durch Transfluenz vergrößerte und vertiefte Verwerfung. Insgesamt zeigt sich ein Bild einer selbständigen Vergletscherung des Belchengebietes mit einer sehr geringen oder kaum vorhandenen Verbindung zum Schauinsland-Feldberg-Massiv.

\section{[The Mount Belchen Region during the Würm Stage]}

Abstract: Primary centres of glaciation besides the Mount Belchen-Heidstein were the districts named Köhlgarten in the southwest and the Breitnauer Kopf in the northwest. During the glacial maximum of Würm stage the ice-masses united with the Wiesetal-glacier in the east. In the western part the glacier-tongues were short in consequence of the high relief gradient; moreover the deliveries are rare. The best preserved cirque is the so called Nonnenmattweiher; but 5 more cirques are known. The documents of retreat of

*) Anschrift des Autors: Dr. G. RaHM, Deichelweiherweg 6, 7800 Freiburg i. Br. ice are badly delivered as is shown in the moraines of the Titisee stade. However it is possible to calculate it by the snowline. The Falkau-Zipfelhof-stade has left behind moraines at several places; the Waldhof-stade is best verified in the village named Multen. During the Feldseestade only the summit of Mount Belchen was ice-capped, and in the cirque Nonnenmattweiher last ice-remains maintained. As glacial features there are many roches moutonnées, Ushaped valleys and wide hollows. The Krinne in the north of Mount Belchen was originally a fault, which was broadenend and overdeepend by transfluence. As a whole the Belchen region makes up an independent glaciation with very few or without relations to the Schauinsland-Feldberg-Massive.

\section{Einführung}

Eines der 5 Großzentren der würmzeitlichen Vergletscherung des Südschwarzwaldes war das bis heute nur selten behandelte Belchengebiet. Die bisher wichtigste Beschreibung dieses Gebietes gab SCHREPFER (1931). Hier soll versucht werden, den heutigen Kenntnisstand darzulegen.

Neben dem Belchen (1414 m) als Hauptvereisungszentrum gab es mehrere weitere Zentren, die mit dem Belchen locker zusammenhängen und von denen kleinere Gletscher ernährt wurden: Nordnordöstlich vom Belchen brachte der Heidstein $(1274 \mathrm{~m}) \mathrm{Zu}$ schüsse zu dem nach $\mathrm{E}$ ablaufenden Gletscher des Belchen und sandte je eine getrennte Zunge nach SW und N; Der Breitnauer Kopf (1121 m), 2,5 km weiter nördlich gelegen, hatte kleine Gletscherzungen nach NE, NW und SW; Südwestlich des Belchen hatte der Köhlgarten $(1224 \mathrm{~m})$ zusammen mit der Sirnitz $(1114 \mathrm{~m})$ nur eine lockere Verbindung zum übrigen Gebiet; von hier gingen Gletscher nach N, W und $\mathrm{S}$ aus. 


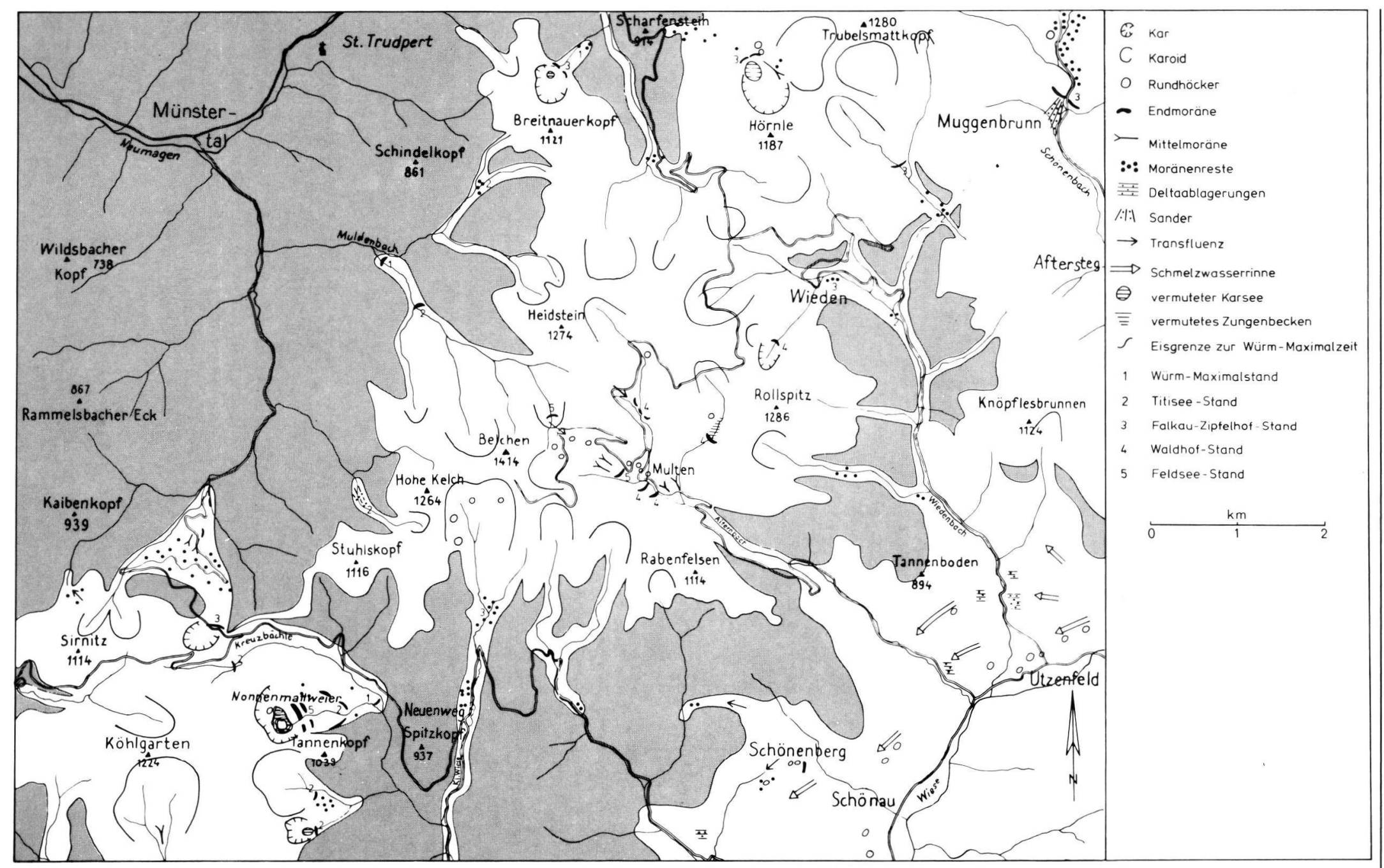




\section{Die Ostseite des Belchen}

Ein langer Gletscherstrom lief vom Belchen nach $\mathrm{E}$ über das Kaltwasser - Untermulten das A i t e $\mathrm{r}$ n $\mathrm{b}$ a ch t a l hinab und vereinigte sich mit dem Wiesegletscher wahrscheinlich schon in der Gegend von Holzinshaus, denn bis so hoch hinauf (etwa 800 $\mathrm{m})$ reichte das Eis des Wiesegletschers (SCHREPFER 1931: 203; GÖLLER 1952: 58 Nr. 108). Dieser Gletscher, in der Hauptsache vom Belchen ausgehend, bekam Zuwachs vom Rübgartenkopf (1246 m) und von weiter westlich her durch den tiefen Einschnitt zwischen Belchen und Rübgartenkopf (P. 1204,8), der als Transfluenz fungierte. Es ist nämlich anzunehmen, daß der Niederschlag auf der Westseite des Kammes so stark war, daß die Eisscheide westlich der Wasserscheide lag, mithin ein Teildes Eises von dort nach $\mathrm{E}$ abfließen konnte. Die Tiefe des Einschnittes ist durch die Erosion des Eises in der Transfluenz zu erklären, wenn auch die Moräne durch späteren Hangschutt überschüttet wurde und nicht aufgeschlossen ist. Auch vom Heidstein (1274 m) her bekam der Gletscher über den Dietschelbach einen kräftigen Zuwachs mit weiterer Ernährung über eine Transfluenz in der Krinne, wo SCHREPFER (1931: 202) Moräne unter postglazialem Hangschutt als möglich ansah, sonst aber in der Krinne einen „Taltorso” sah. Zweifellos ist die Krinne so tief eingeschnitten, weil hier die südliche Verwerfung des Münstertal-Grabens hindurchgeht, und diese zerrüttete Zone wurde durch das Eis in der Transfluenz noch vertieft und später mit Hangschutt überschüttet.

Über den Hintergrundbach lieferte der Hoh Tannen $(1248,7 \mathrm{~m}), 1,5 \mathrm{~km}$ ostsüdöstlich des Heidstein, Eis zum Aitern-Gletscher. Nur die drei großen vereinigten Eisströme vermochten den Weg nach SE bis zum Wiesetal zu bewältigen. Dort, wo die drei Hauptströme vom Belchen, Heidstein und Hoh Tannen zusammenliefen, im Raume Untermulten, ist durch die erheblich vermehrte Eismenge das Tal als weite Wanne ausgehobelt worden, in welche heute die drei Bäche mit Talstufen, der Dietschelbach gar mit einem Wasserfall einmünden. SCHREPFER (1931: 202) sagt, daß sich ,in der Landschaft um Multen . . . die meisten der im Schwarzwald auftretenden Glazialformen an guten Beispielen studieren" lassen. Auch berichtet er von „Grundmoräne in außergewöhnlicher Mächtigkeit". Es sind aber auch mehrere Moränenreste erhalten, die von einem größeren Rückzugsstand zeugen.

Zwei Endmoränenwälle queren in 1000 bis $1020 \mathrm{~m}$ Höhe oberhalb des Wirtshauses von Multen das Tal. Hier fanden die Gletscher vom Dietschelbach, dem Rübgartenbächle und vom Gfällwasserbächle ihr gemeinsames Ende, als die Schneegrenze in etwa 1250 m Höhe lag. Das entspricht dem Waldhof-Stand
(PlatZ 1893; HanTKe \& RAHM 1976). Zu dieser Zeit endete der Hintergrundbach-Gletscher in 1070 bis $1075 \mathrm{~m}$ Höhe, dort ist ebenfalls ein recht deutlicher Endmoränenwall erhalten, hinter welchem das vermoorte Gelände heute noch das ehemalige Zungenbecken anzeigt.

Die Moränenreste in Untermulten und beim Forsthaus sind als Mittelmoränen zwischen DietschelbachRübgarten-Gfällwasser-Gletscher und dem Hintergrundbach-Gletscher zu deuten aus einer Zeit, als die Gletscher noch gemeinsam weiter talab liefen. Vermutlich gehören sie zum Titisee-Stand, der aber sonst im Aiterntal nicht weiter dokumentiert ist. Der Gletscher muß zu dieser Zeit etwa bis Holzinshaus gereicht haben, der Wiesegletscher war aber schon weit über Schönau zurückgeschmolzen.

Oberhalb der Gefällstufe ist zwischen dem Gfällwasserbächle und dem Rübgartenbächle bei der Bergwacht in $1070 \mathrm{~m}$ Höhe ebenfalls ein Endmoränenwall vorhanden, der dem äußeren Feldsee-Stand (Feldseemoos-Stadium nach HANTKE \& RAHM 1976: 298) angehört. Nur wenig weiter oberhalb verlaufen zwei Mittelmoränenwälle etwa parallel zum Rübgartenbächle zwischen diesem und dem Gfällwasserbächle.

Die Hauptmasse des Eises zur Ernährung des Aiternbach-Gletschers kam vom Belchen direkt über Kaltwasser und Gfällwasserbächle herunter. Trotzdem sind im oberen Teil dieses Tales keine morphologischen Merkmale eines Gletschertales zu erkennen. Den Bereich des Kaltwasser östlich vom Belchenhaus sieht SCHREPFER (1931: 208, Karte) als Kar an. Da hier jedoch jegliche Andeutung eines ebenen Bodens und steile Seitenwände fehlen, kann nur von einem Kartrichter oder Karoid gesprochen werden (unter Vermeidung des Begriffes "Zirkusschluß”, entsprechend LIEHL 1982: 39). Ein Teil des Eises aus dem Kaltwasser floß auch nach NE in das Rübgartenbächle-Tal, was von einer Reihe von Rundhöckern und Talstufen bezeugt wird. Die Kerbe zwischen Belchen und Rübgartenkopf, die zur Zeit der Würm-Maximalstände als Transfluenz in W-E-Richtung diente, hat in späterer Zeit für den Eistransport keine Rolle mehr gespielt, sie ist deshalb bereits frühzeitig mit Hangschutt erfüllt worden.

Der obere Teil des Dietschelbach-Tales ist durch den Gletscher vom Heidstein zu einem schönen Trogtal geformt worden. In Obermulten sind in 1060 bis 1070 m Höhe deutliche Endmoränenreste vorhanden, die dem Waldhof-Stand zuzurechnen sind wie diejenigen im Hintergrundbach-Tal. Im obersten Teil des Dietschelbach-Tales zeugt ein in $1140 \mathrm{~m}$ Höhe gelegener Rundhöcker von der Erosionsarbeit des Gletschers. Auch der Dietschelbach-Gletscher begann in einem Kartrichter. 
Der Gletscher im W i e d e $\mathrm{n} \mathrm{b}$ a $\mathrm{ch}-\mathrm{T}$ a $\mathrm{l}$ erreichte in der Würm-Maximalzeit ebenfalls den Wiesegletscher. SCHREPFER (1931: 204) nahm das Ende des Wiedenbach-Gletschers bei der Säge in $760 \mathrm{~m}$ Höhe an, weil weiter unterhalb zwischen Säge und Königshütte Glazialspuren fehlen. Auf Grund der Schneegrenze zur Würm-Maximalzeit in $950 \mathrm{~m}$ Höhe (EExposition) müßte der Gletscher allerdings bis unter $700 \mathrm{~m}$ hinabgegangen sein, zumal vom Hohtannwald (Hoh Tannen 1248, 7 m, Rollspitz 1236,1 m) über Mittelbach und Warbach kräftiger Eiszuwachs kam. Auch von der Nordseite vom Hörnle (1187 m) über Rütte, und über Ungendwieden floß Eis zum Wiedenbach-Gletscher. Die Tatsache, daß unterhalb der Säge keine Glazialspuren vorhanden sind, ist dadurch zu erklären, daß das Eis des großen Wiese-Gletschers nach GIERMANN (1959: 41) bis auf etwa 850 m reichte, es drückte also hoch in das Wiedenbachtal hinauf und staute diesen Gletscher, der dadurch in seiner Bewegung gehemmt wurde und keine Glazialspuren hinterlassen konnte.

Für den Gletscher vom Rüttener Grund (1109,3 m) nahm SCHREPFER (1931: 204-205) das Ende unterhalb von Ungendwieden auf etwa $955 \mathrm{~m}$ an. Hier gilt das gleiche wie für den Wiedenbach-Gletscher: Im Maximum muß der Gletscher weiter gegangen sein und sich mit dem Wiedenbach-Gletscher vereinigt haben. Der Stau am Eis des Wiesetales hat Bewegung und entsprechende Erosion verhindert.

Die direkte Verbindung der Gletscher im Aiternbachtal und im Wiedenbachtal mit dem Wiese-Gletscher hat im Maximum der Würmeiszeit sicherlich nicht sehr lange bestanden. Schon bei dem ersten geringen Rückschmelzen des Eises ging die Verbindung verloren und es bestand zwischen dem Wiese-Eis und den beiden Gletschern eine eisfreie Zone. Vor dem Wiese-Eis wurde das Schmelzwasser beider Täler in Eistauseen aufgefangen, in welche die Schmelzwässer des Wiese-Gletschers über eigene Schmelzwasserrinnen hineinflossen und dort Deltakiese und -sande ablagerten. Reste davon sind heute noch in der Sandgrube im Gewann Kessel im Wiedenbachtal und bei der neuen Siedlung südöstlich Aitern unter dem Rollsberg vorhanden. GIERMANN (1959: 41-44) hat das Abtauen des Eises in diesem Raum in 4 Phasen beschrieben. Es ist natürlich auch zu dieser Zeit mit kurzen Wiedervorstößen zu rechnen, denn die Deltaablagerungen sind mit Moräne überdeckt. Aứh über den Utzenbach und den Gschwenderbach wurde noch vom Knöpflesbrunnen (1123,8 m) Eis hinzugeliefert.

Zur Zeit des Titisee-Standes erreichte der Wiedenbach-Gletscher, in welchem Teile vom Wiedener Eck, über Mittelbach und über Warbach zusammenliefen, noch das untere Ende des Ortes Wieden, wo SCHREPFER
(1931: 204) bei der Säge in $760 \mathrm{~m}$ Höhe das Ende des Gletschers zur Maximalzeit annahm. Zur Titisee-Zeit konnte der Gletscher nicht weiter reichen, weil von $\mathrm{N}$ her über den Spitzdobel bereits Schmelzwasser abfloß, das möglicherweise sonst weiter vorstoßendes Eis nicht zuließ. Im Ungendwiedenbachtal erreichte der Gletscher, der vom Rüttenergrund, vom Hüttbachergrund und vom Meßlergrund gespeist wurde, nur noch etwa 900 m Höhe, gerade den oberen Beginn des Spitzdobels.

Das Eis im Gschwenderbach- und Utzenbachtal war zur Titiseezeit nur noch in den obersten Nischen am Südhang des Knöpflesbrunnen vorhanden.

Der Falkau-Zipfelhof-Stand (HANTKE \& RAHM 1976) ist im Wiedenbachtal durch einen Moränenrest in $875 \mathrm{~m}$ Höhe nahe der Neßlerhäuser dokumentiert (SCHREPFER 1931: 204), wo noch gerade die drei Gletscherzungen vom Wiedener Eck, Mittelbach und Warbach zusammenkamen. Oberhalb Ungendwieden gehört hierher die Moräne beim Kellerhaus in $960 \mathrm{~m}$ Höhe. Auch hier dürften gerade noch die drei Eiszungen vom Rüttenergrund, Hüttbachergrund und Neßlergrund zusammengetroffen sein.

Der Waldhof-Stand ist nur im Warbacher Tal dokumentiert, wo im Fuchsgrüble ein ,hübsches kleines Kar" (SCHREPFER 1931: 204) durch einen Moränenwall abgeschlossen wird. In allen anderen Tälern sind keine höher liegenden Glazialrelikte mehr zu finden. Auch die Herkunft der Gletscher ist nur durch die Kartrichter festzustellen. Im ganzen Einzugsgebiet des Wiedenbaches gibt es nur das eine gut ausgebildete Kar im Fuchsgrüble in NE-Exposition unter dem Rollspitz (1236,1 m).

Der B ölle n b a ch - Gletscher beginnt am Rosenfelsen am Südosthang des Belchen; er wurde von dem vom Belchen nach $S$ ziehenden Gebirgskamm noch miternährt. Weiterer Zuwachs kam über den Gletscher des Böllenbächletales. Im Bereich des Zusammenflusses beider Gletscher bildete sich, beginnend mit einer Konfluenzstufe, ein weiter Talkessel aus, an dessen oberem Rand Oberböllen liegt. Nach SCHREPFER (1931: 201) ist der tiefste bekannte Moränenaufschluß in diesem $\mathrm{Tal}$ beim heutigen P. 672,1 (bei SCHREPFER P 674). In dieser Gegend ist auch das Ende des Böllenbach-Gletschers anzunehmen.

Durch ein Unwetter am 22. 2. 1970 ist am Südostende der Rimshalde in 660 m Höhe ein Aufschluß entstanden, der Moräne über Deltasanden zeigte. Diese gehören nicht mehr zum Böllenbach-Gletscher, sondern sie sind durch den Wiese-Gletscher entstanden: über Schönenberg drang Wiese-Eis in Transfluenzen in das Tal des Lehbächle ein, später floß Schmelzwasser über diesen Weg und schüttete Deltasande in einem See 
auf, der im Böllenbachtal vor dem Wiesetal-Eis gestaut wurde. Auch hier zeigt die darüberliegende Moräne an, daß es Wiedervorstöße des Wiese-Gletschers gab.

Auch im Wildböllenbachtal lag im obersten Teil ein sehr kleiner Gletscher, von dem leider in dem Waldgebiet kaum Spuren zu erkennen sind. Bei Wildböllen selbst ist der Wiese-Gletscher über das Sägeneck in das Tal eingedrungen, wie GÖLLER (1952: 62, Nr. 144 u. 145) zeigen konnte, denn die Moränen sind eindeutig diesem Gletscher zuzuschreiben. Es zeigt sich, daß auch hier der Wiese-Gletscher noch eine Eishöhe von gut $850 \mathrm{~m}$ hatte. Schon im Titisee-Stand ist der Gletscher nicht mehr bis Oberböllen gekommen. Weiter oberhalb fehlen jegliche Glazialspuren sowohl im Böllenbachtal (GW Eibenwald) als auch im Böllenbächletal (GW Breitmoos). Die Täler beginnen mit Kartrichtern.

\section{Die Südseite des Belchen}

Der Gletscher der Kle in e n (Belche n-) W i e s e floß direkt vom höchsten Belchenbereich nach $\mathrm{S}$ ab. Trotz des bedeutenden Liefergebietes sind nur sehr wenige Glazialspuren in diesem Tal vorhanden. Entsprechend der Schneegrenze im Würm-Maximum müßte der Gletscher bis auf $500 \mathrm{~m}$ hinab gereicht haben, wie es auch beim großen WieseGletscher und anderen bedeutenden Gletschern im Schwarzwald der Fall war. Tatsächlich befindet sich zwischen Höll und Langensee, gerade in $500 \mathrm{~m}$ Höhe, ein Wall, der allerdings mangels Aufschluß nicht klar zu deuten ist. Trotzdem ist hier das Gletscherende für das Würm-Maximum anzunehmen, auch wenn SCHREPFER (1931: 192) der Ansicht ist, daß es sich bei dem Wall um eine Vermurung handele. Andere Beobachtungen sprechen für ein Ende des Gletschers kurz unterhalb Bürchau, wo bei den Kastelhöfen das Tal enger wird. In jedem Fall muß der Gletscher entgegen der Meinung SCHREPFER's (1931: 192) weit über Neuenweg nach $\mathrm{S}$ vorgedrungen sein.

Bei der Kirche von Neuenweg ragt ein Sporn in das Tal hinein. SCHREPFER (1931: 192) nennt diesen die „Kirchterrasse” und sah dort „,Moräne an den Fels angeklebt". Heute ist im angeschnittenen Hang beim Friedhof nur verwittertes Paläozoikum zu erkennen, das leicht Anlaß zu Verwechslungen mit Moräne geben kann. Doch auch hier muß, der Morphologie nach zu urteilen, ein längerer Gletscherhalt gewesen sein, der dem Titisee-Stand zuzuschreiben ist.

Bei den Belchenhöfen liegt in 790-830 m Höhe Grundmoräne mit einzelnen großen Blöcken. In diesem Bereich ist der Falkau-Zipfelhof-Stand zu erwarten.
Weitere Rückzugsstände sind im Tal der Kleinen (Belchen) Wiese nicht zu erkennen. Das Tal beginnt mit einem großartigen Kartrichter, dessen steile Rückwand vom Belchen mit Blick auf Neuenweg besonders eindrucksvoll erscheint. Der Kartrichter ist vom Hochfelsen - Südhang des Belchen - Hohe Kelch $(1263,7 \mathrm{~m})$ und dem nach $\mathrm{S}$ anschließenden Heideck $(1131,5 \mathrm{~m})$ eingefaßt. Einzelne Felspartien sind vom Eis rundgeschliffen, der Heideckfelsen und andere nicht benannte Felsbuckel sind Rundhöcker.

\section{Die West- und Nordseite des Belchen}

Auf der West- und Nordseite des Belchen fallen eine ganze Reihe von Tälern steil $a b$; sie beginnen alle in Kartrichtern, von denen aus in der Würm-Eiszeit Gletscher diese Täler hinabzogen. Durch die besonders hohe Reliefenergie in diesem Bereich (Höhenunterschied $780 \mathrm{~m}$ auf $3 \mathrm{~km}$ Horizontaldistanz im Starkenbrunnental $=26 \%, 900 \mathrm{~m}$ auf $2,5 \mathrm{~km}$ im Knappengrund $=36 \%$ ) sind nur sehr wenige Dokumente der ehemaligen Vergletscherung erhalten geblieben.

Im $\mathrm{M} \mathrm{u} \mathrm{l} \mathrm{d} \mathrm{e} \mathrm{n} \mathrm{b} \mathrm{a} \mathrm{c} \mathrm{h} \mathrm{t} \mathrm{a} \mathrm{l}$ sind knapp oberhalb des Zusammenflusses von Muldenbach und den von der Nordseite des Belchen kommenden Flüssen nordwestlich vom Kaltwasser in 490 m Höhe Moränenreste erkennbar. Die Ausdehnung des Gletschers vom Knappengrund mit Zuflüssen von der Südwestseite des Heidstein (1274,3 m) und von der Nordseite des Belchen entspricht bei einer Schneegrenze von $950 \mathrm{~m}$ gerade dem Maximal-Stand.

Weitere Moränenreste liegen am Zusammenfluß von Knappengrund und Kaltwasser, die dem TitiseeStand angehören.

In den von dort steil nach oben führenden Tälern sind keine deutlichen Glazialspuren mehr vorhanden. Der Talschluß im Knappengrund ist ein ebenso großartiger Kartrichter, wie jener der Kleinen (Belchen) Wiese, nur ist er hier im Wald nicht so deutlich sichtbar. In dem von der Krinne nach W führenden Tal lag ebenfalls Gletschereis, das sowohl vom Rübgartenkopf $(1246,1 \mathrm{~m})$ als auch vom Heidstein $(1274,3 \mathrm{~m})$ ernährt wurde. Durch den von W herangeführten Niederschlag war dieses Tal ebenso wie das Rosenbächletal wenigstens zur Würm-Maximalzeit so hoch mit Eis aufgefüllt, daß ein Teil des Eises über die Krinne zum Dietschelbachtal und vom Rosenbächle zum Rübgartenbächletal in Transfluenzen abfloß.

Im Rosenbächle beschreibt SCHREPFER (1931: 190) in 1220 m Höhe eine Moräne, die nur knapp 30 m unter der Transfluenz wahrscheinlich in den Feldsee-Stand $\mathrm{zu}$ stellen ist. 


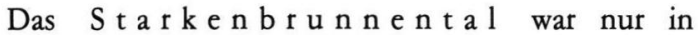
seinem obersten Teil vergletschert. Von P. 714 an aufwärts ist das Tal auffallend breiter, als in seinem unteren Teil. Hier ist das Gletscherende anzunehmen. SCHREPFER (1931: 189) nahm das Ende des Gletschers in $760 \mathrm{~m}$ Höhe an, wo er eine „Ufermoräne mehrere $100 \mathrm{~m}$ talaufwärts" verfolgen konnte. Diese mächtigen Aufschüttungen betrachte ich eher als einen Sander, der zu einem in etwa $800 \mathrm{~m}$ Höhe anzunehmenden Rückzugshalt gehört, der dem Titisee-Stand zuzurechnen ist.

Im W ölflin s g r u n d gab es keine Vergletscherung. Der heutige Bach entspringt am Nordwesthang des Stuhlskopf $(1116 \mathrm{~m})$, der nur wenig über die damalige Schneegrenze hinausragte. Ab etwa $700 \mathrm{~m}$ aufwärts liegt im Wölflinsgrund ein bis zu $4 \mathrm{~m}$ mächtiger Hangschutt, der wegen seiner Mächtigkeit nicht alleine in der Nacheiszeit entstanden sein kann, sondern auch zur Würm-Eiszeit im damaligen Periglazialgebiet gebildet wurde.

\section{Das Köhlgarten-Gebiet}

Ein zweites großes Zentrum der Vergletscherung war neben dem Belchen-Heidstein-Gebiet der Köhlgarten $(1224 \mathrm{~m})$, der sich als mächtig aufragender Bergrücken vom Spähnplatz $(1053,1 \mathrm{~m})$ bis zum Tannenkopf $(1039 \mathrm{~m})$ in fast $3 \mathrm{~km}$ Länge in W-E-Richtung hinzieht.

An seiner Nordostseite liegt der bekannte $\mathrm{N}$ o $\mathrm{n}$ $\mathrm{n}$ e $\mathrm{n} \mathrm{m}$ a $\mathrm{t} \mathrm{t}$ w e i h e $\mathrm{r}(913 \mathrm{~m})$, ein besonders gut ausgebildetes Kar mit einer rund $100 \mathrm{~m}$ hohen Felsenrückwand. Entstanden ist dieser Kessel durch glaziale Erosion in einer Quellmulde, die bereits vorher bestand. Der Gletscher, der von hier aus seinen Weg nahm, hat allerdings trotz der großartigen Kar-Nische nicht besonders weit gereicht. Die Häuser von Vorderheubronn stehen auf einem Moränenwall, der zum Maximal-Stand gehört. Hier flossen auch gerade noch der Weiherbach-Gletscher, aus dem Nonnenmattweiher kommend, und $\operatorname{der} \mathrm{Kr}$ e u z b ä c h le Gletscher zusammen, der sowohl vom Nordhang des Köhlgarten als auch vom Südhang des Weiherkopf $(1143,3 \mathrm{~m})$ ernährt wurde. Das Kreuzbächletal ist über Hinter- und Mittel- bis nach Vorderheubronn auffallend breit. Dies ist allerdings nicht alleine auf glaziale Erosion zurückzuführen, sondern hier stehen in der sog. Kulmzone weichere Gesteine an, die die sanften Hänge des Tales hervorrufen. Unterhalb Vorderheubronn, außerhalb der würmzeitlichen Vergletscherung, biegt das Tal nach $\mathrm{S}$ ab und wird sehr eng. Bezeichnenderweise heißt der Bach auch vom Zusammenfluß von Kreuzbächle und Weiherbach „Klemmbach", der sogar mit einem kleinen Wasserfall am
Anfang seinen Lauf nimmt. Hier ist die westlichste Anzapfungsstelle des ehemaligen nach SCHREPFER (1931: 192) W-E-gerichteten Flußlaufes vom Nonnenmattweiher über das Eck - Schanze - Sägeneck zur Wiese.

Geht man von Vorderheubronn den Weiherbach aufwärts, so trifft man bei der Wegkehre in $840 \mathrm{~m}$ Höhe wiederum Moräne an, die sich vom Nordhang des Tannenkopfes und vom Dürsberg-Südhang bis dort hinabzieht. Es handelt sich um den Rückzugshalt des Titisee-Standes.

Der Nonnenmattweiher selbst wird durch mindestens zwei Moränenwälle abgeriegelt, von denen der innere Wall auffallend breit ist. Er könnte von zwei eng beieinander liegenden Wällen gebildet sein. Auch diese Moränen ziehen vom Dürsberg herunter, sind aber südlich des Weiherbaches am Hang des Tannenkopf auch noch deutlich zu erkennen. SCHREPFER (1931: 193) bezeichnet sie als „eine der schönsten Moränen des Schwarzwaldes" und gibt ihre Höhe mit $20-25 \mathrm{~m}$ an. Rein rechnerisch sollte man hier den Falkau-Zipfelhof-Stand erwarten, jedoch spricht der 2-3-fache Moränenwall eher für den Feldsee-Stand. In der tiefen Nische konnte sich der Gletscher sicher entsprechend lange halten. Am Feldsee selbst ebenso wie in Hintermenzenschwand und an anderen Stellen ist dieser Stand auch jeweils durch einen 3-fachen Wall dokumentiert.

Im Kreuzbächletal wurde bei Hinterheubronn durch Häuserbau Moräne in $890 \mathrm{~m}$ Höhe aufgeschlossen, die dem Titisee-Stand zuzurechnen ist.

Auf der Nordseite des Köhlgarten sind keine weiteren Glazialspuren vorhanden, jedoch auf seiner Ost- und Südseite.

Südlich des Nonnenmattweiher entwässert das $\mathrm{T}$ a n n e n b ä c h l e den östlichen Köhlgarten aus dem Peterlegraben und dem Seile $\mathrm{m}$ o o $\mathrm{s}$ nach $\mathrm{E}$. Während im Peterlegraben nur ein großer Kartrichter von der ehemaligen Vergletscherung zeugt, ist das Seilemoos ein gegenüber dem Nonnenmattweiher zwar wesentlich kleineres, aber doch sehr deutliches Kar mit einem vermoorten Karboden in $885 \mathrm{~m}$ Höhe. Die Gletscher aus diesen beiden Quellästen haben das Haupttal, den Klemmbach, auch im Würm-Maximum nicht erreicht, sie haben nach SCHREPFER (1931: 194) kurz unterhalb ihres Zusammentreffens in $750 \mathrm{~m}$ Höhe ihr Ende gehabt. Weiter oberhalb ist das Gewann „Auf dem Zimmerplatz" mit Grundmoräne mit vielen Geschieben überdeckt. Im Peterlegraben ist eine Endmoräne in knapp $850 \mathrm{~m}$ Höhe zu erkennen, die dem TitiseeStand zuzuordnen ist. Das Seilemoos-Kar wird nach E von einer Moräne abgeschlossen (ROSER 1899: 15), die ebenfalls zum Titisee-Stand gehört. 
Nach S entwässern den Köhlgarten der $\mathrm{K}$ ü h l e $\mathrm{n}$ $\mathrm{bronnerbach}$ und das $\mathrm{Fischenber-}$ g e r b ä c h l e. Im Sägmatt, dem Zusammenfluß beider Bäche (außerhalb der Karte), liegt in $670 \mathrm{~m}$ Höhe eine mächtige Endmoräne (ROSER 1899: 15), die das gemeinsame Ende beider Gletscher zur Würm-Maximalzeit dokumentiert. Sonst sind die Glazialdokumente in beiden Tälern recht spärlich. SCHREPFER (1931: 194) beschreibt in dem glazialen Kessel oberhalb Kühlenbronn eine Endmoräne in 890-900 m Höhe, die allerdings eher als Mittelmoräne zwischen den beiden Quellästen des Kühlenbronnerbaches anzusehen ist. Sie ist deshalb auch keinem Rückzugsstand zuzuordnen. Beide genannten Täler beginnen in Kartrichtern.

\section{Das Sirnitz-Weiherkopf-Gebiet}

Nordwestlich des Köhlgarten bildete die Sirnitz $(1114 \mathrm{~m})$ mit dem Weiherkopf (1143,3 m) ein weiteres eigenes Vergletscherungsgebiet. Die Zulieferung von dort zum Kreuzbächle-Gletscher nach Heubronn wurde bereits erwähnt.

Jenseits des Sirnitz-Sattels (Kreuzweg) gelangt man in das nach $W$ entwässernde $\mathrm{K} \mathrm{le} \mathrm{m} \mathrm{m} \mathrm{b}$ a $\mathrm{h} \mathrm{t} \mathrm{a} \mathrm{l}$, in dem bisher noch keine Spuren einer Vergletscherung gefunden wurden. Trotzdem ist anzunehmen, $\mathrm{da} \beta$ auch dieses $\mathrm{Tal}$ vergletschert war. Zumindest die obere flache Wanne dieses Tales bis etwa $865 \mathrm{~m}$ hinab war von Gletschereis erfüllt. In der von dort abwärts einsetzenden engen Schlucht, die durch die rückschreitende Erosion vom tiefgelegenen Rheintal geformt wurde, sind alle Dokumente zerstört worden.

Deutlicher sind die Spuren der Vergletscherung auf der Nordseite der Sirnitz (SCHREPFER 1931: 188-189). Das weite B e c ke n v o n $\mathrm{M}$ ü n $\mathrm{st}$ e $\mathrm{rha} \mathrm{ld} \mathrm{e} \mathrm{n}$ ist von Grundmoräne erfüllt und einige Rücken deuten Endmoränen an. Das gemeinsame Ende der Gletscher im S i r n it z g r u n d und $\mathrm{Halde} \mathrm{n} \mathrm{b} \mathrm{a} \mathrm{h} \mathrm{t} \mathrm{a} \mathrm{l} \mathrm{lag} \mathrm{bei} \mathrm{Langenbach} \mathrm{in}$ etwa $570 \mathrm{~m}$ Höhe. Dort sind heute einige parallel zum Talverlauf längliche Rücken zu erkennen, die durch die Zerschneidung der Täler diese Form bekamen. Im Haldenbach fand SCHREPFER (1931: 188) als deutliches Zeichen einer Vergletscherung einen "2 cbm großen prachtvoll geschliffenen Block".

Der Haldenbach-Gletscher begann im nach NE gerichteten Kar unter dem Weiherfelsen, das heute noch einen deutlichen Karboden unter den steilen Felswänden in $900 \mathrm{~m}$ Höhe hat. Abgeschlossen wird dieser Karboden durch einen Moränenwall im Bereich der heutigen Fahrstraße auf dem Parkplatz. Diese Moräne dürfte zum Falkau-Zipfelhof-Stand gehören; es ergibt sich daraus, daß die Vergletscherung hier nur bis zu dieser Zeit andauerte.

Im Sirnitzgrund sind bisher keine höher liegenden Glazialdokumente bekannt geworden, jedoch beschreibt SCHREPFER (1931: 189) „zahllose Geschiebe” bei der Kälbelescheuer, die ein Überfließen dieses Sattels durch den Gletscher anzeigen, der dann noch bis etwa auf $900 \mathrm{~m}$ Höhe das $\mathrm{R} \mathrm{a} \mathrm{m} \mathrm{m} \mathrm{e} \mathrm{l} \mathrm{s} \mathrm{b} \mathrm{a} \mathrm{c} \mathrm{h} \mathrm{t} \mathrm{a} \mathrm{l}$ hinabfloß.

\section{Das Gebiet um den Breitnauer Kopf}

Nördlich vom Belchen-Heidstein-Gebiet war ein weiteres Zentrum der Vergletscherung am Breitnauer Kopf $(1120,9 \mathrm{~m})$. Auf seiner Südwestseite lief ein Gletscher den $\mathrm{K}$ a i b e $\mathrm{n}$ g r u n d hinab und vereinigte sich noch gerade mit einer aus dem Kartrichter des $\mathrm{Holzschlag}$ am Nordwesthang des Heidstein kommenden Gletscherzunge. Eine Endmoräne ist nicht vorhanden (SCHREPFER 1931: 189), jedoch Reste von Grundmoräne, die in der Talweite im Oberlauf des Muldenbaches bei P. 646,6 und 677,4 noch ziemlich mächtig (bis $1,5 \mathrm{~m}$ ) erhalten ist. Hier kann auch der Rückzugshalt des Titisee-Standes vermutet werden.

Ein schönes Kar, das SCHREPFER (1931: 187-188) als Zwillingskar bezeichnet, liegt auf der Nordwestseite des Breitnauer Kopfes im $\mathrm{K}$ üs te l w a ld mit einem Karboden in $930 \mathrm{~m}$ Höhe. Der Gletscher reichte im Würm-Maximum bis zum Weiler „Hinteres Elend" in etwa $700 \mathrm{~m}$ Höhe. Das Küstelwaldkar selbst ist nach NE von einem Moränenwall abgeschlossen, der zum Falkau-Zipfelhof-Stand gehören dürfte. Auch hier hat die Vergletscherung also nur bis zu dieser Zeit gedauert.

Das Küstelwaldkar gehört bereits zum Einzugsgebiet des $\mathrm{St} \mathrm{a} \mathrm{m} \mathrm{p} \mathrm{fe} \mathrm{bä} \mathrm{ch} \mathrm{le,} \mathrm{das} \mathrm{oberhalb} \mathrm{Unter-}$ neuhof in einer weiten Mulde aus vier Quellbächen entspringt. Alle vier Quellbäche beginnen in Kartrichtern, so daß anzunehmen ist, daß die weite Mulde mit Gletschereis gefüllt war. Bei Unterneuhof sind in $865 \mathrm{~m}$ Höhe noch geringe Reste von Moräne zu erkennen, hier nehmen sowohl SCHREPFER (1931: 187) als auch GIERMANN (1959: 15) die Grenze der Vergletscherung an. STEINMANN (1896: 203-204) vermutet das Gletscherende beim Elendfelsen in $600 \mathrm{~m}$ Höhe. Diese letztere Auffassung dürfte eher den Tatsachen entsprechen, denn auch dann kann die enge Schlucht zwischen Unterneuhof und Stampfe durch subglaziale Schmelzwässer fluviatil, wie von SCHREPFER (1931: 187) gefordert, bzw. glazifluvial entstanden sein.

Von der Ostseite mündet der $\mathrm{G} \mathrm{l}$ a s h of b a c h bei Stampf in das Stampfebächle. Auch dieses Tal war 
im Oberlauf vergletschert, wie es SCHREPFER (1931: 187) und GIERMANN (1959: 16-17) bereits beschrieben. Das Ende des Gletschers zur Würm-Maximalzeit lag bei der Straßenkehre am Scharfenstein in $795 \mathrm{~m}$ Höhe. Dies wird durch immer wieder auftretende Grundmoräne oberhalb dieses Punktes belegt. Die beiden Quelläste des Glashofbaches kommen aus dem Scheibenmossgebiet, einem Kartrichter, und dem Weiherwald, in dem das sehr gut ausgebildete $\mathrm{Gla} \mathrm{sh} \mathrm{of} \mathrm{k} \mathrm{a} \mathrm{r} \mathrm{mit} \mathrm{einem} \mathrm{deutlich} \mathrm{ausgebildeten}$ Karboden „Im Weiher” in 970 m Höhe sitzt. Dieses nach NW gerichtete Kar wird durch eine Endmoräne abgeschlossen, die dem Falkau-Zipfelhof-Stand anzugehören scheint. Auch hier dauerte die Vergletscherung demnach bis zu diesem Rückzugsstand. Der nach SW gerichtete Kartrichter im Scheibenmoos ist wohl schon früher eisfrei geworden. Zwischen den beiden Quellbereichen befindet sich eine deutliche Zwischenmoräne, und letztlich beweisen zwei schöne Rundhöcker in $1000 \mathrm{~m}$ Höhe die ehemalige Vergletscherung dieses Gebietes.

\section{Schlußbetrachtung}

Die Zusammenfassung aller Glazialdokumente ergibt das Bild einer auch in der Würmeiszeit noch bedeutenden Vergletscherung des weiteren Belchengebietes. Dem Hauptzentrum Belchen-Heidstein folgt als nächst wichtiger Bereich das Köhlgartengebiet. Weitere Zentren sind Sirnitz mit Weiherkopf, Breitnauer Kopf und Hörnle-Trubelsmattkopf. Im E reicht der Einzugsbereich des Wiese-Gletschers, der vom Feldberg kommt, in das Gebiet hinein.

$\mathrm{Zu}$ den glazialen Erosionsformen zählen als auffallendste Erscheinungen die Kare, von denen der Nonnenmattweiher als einziger noch mit Wasser gefüllt ist. Weitere gut ausgebildete Kare sind das Seilemoos südlich des Nonnenmattweiher, wo der ehemalige See vermoort ist; weiterhin das Küstelwaldkar und das Glashofkar im Breitnauer Kopf-Gebiet, wo ebenfalls die ehemaligen Seen noch nachweisbar sind; es folgen das Fuchsgrüble am Nordhang des Rollspitz und das Weiherfelsenkar am Osthang des Sirnitz-Massivs. Außer diesen 6 Karen sind alle übrigen Gletscheranfänge lediglich Kartrichter. Besonders um den Belchen selbst, von dem allseits Gletscher zu Tal flossen, befindet sich kein einziges voll ausgebildetes Kar. Hier war die Gletscherbewegung in den steilen Tälern offenbar zu schnell, so daß keine Zeit zur vollen Ausbildung der Kare blieb.

Rundhöcker treten besonders gehäuft im oberen Teil des Rübgartenbächle auf; sie kommen aber auch sonst immer wieder vor. Zu erwähnen ist der von der Straße
Wiedener Eck - Multen gut sichtbare Rundhöcker bei der Straßenkehre im Geläubwald im Dietschelbachtal. Auch die Felspartien in dem großen Zirkusschluß am Südhang des Belchen, der Quellmulde der Kleinen (Belchen) Wiese, sind zu Rundhöckern umgearbeitet.

Glaziale Trogtäler zeigen am deutlichsten die Quelltäler vom Aiternbach (Rübgartenbächle, Dietschelbach und Hintergrundbach), aber ebenso zeigen diese Form das Wiedenbachtal, das Tal der Kleinen (Belchen) Wiese und das Kreuzbächletal bei Heubronn. Auf der Westseite des Belchen ist das Becken von Münsterhalden und im $\mathrm{N}$ das Becken von Neuhof deutlich glazialer Entstehung.

Glaziale Ablagerungen sind um den Belchen spärlicher vertreten, weil durch die große Reliefenergie die postglaziale Erosion viel zerstört hat. Aber besonders in den trogförmigen Oberläufen auf der Ostseite des Belchen - Heidstein - Hörnle - Trubelsmattkopf - Kammes ist noch genügend Grundmoräne vorhanden, ebenso im Kreuzbächletal bei Heubronn und im Becken von Münsterhalden. Jedoch ist auf der Westseite des genannten Kammes die Grundmoräne nur noch in allerletzten Resten vorhanden. Endmoränen, die als Wallform quer über das Tal gehen, sind nur selten erhalten. Sie fehlen auf der Ostseite des Kammes für den Würm-Maximalstand gänzlich, weil die Gletscher mit dem großen, vom Feldberg kommenden Wiese-Gletscher zusammenliefen. Nur in den kleinen Seiten und Quelltälern sind noch einige Endmoränen der Rückzugs-Stände erhalten. Am deutlichsten sind sie um Multen und im Hintergrundbachtal, wo oberhalb der Endmoräne noch ein stark versumpftes Zungenbecken erhalten ist. Mächtigere Endmoränen sind vom Weiherbach-Gletscher, der aus dem Kar des Nonnenmattweiher kam, erhalten. Im übrigen findet man häufiger an den Karen und Kartrichtern noch eine Abriegelung durch Endmoränen, die höchstwahrscheinlich verschiedenen Rückzugsständen zuzuordnen sind.

Mittelmoränen sind sehr selten, so bei Untermulten bei der Mündung des Hintergrundbaches in den Aiternbach, am Südhang des Köhlgarten im Kühlenbronnertal und möglicherweise im Becken von Münsterhalden.

Auf der Westseite des genannten Kammes ist lediglich ein Maximum-Stand im Muldenbachtal angedeutet und dort und in weiteren Tälern Reste von Rückzugsständen. Sonst hat die scharfe Erosion viel zerstört. 


\section{Schriftenverzeichnis}

GiermanN, G. (1959): Die würmzeitliche Vergletscherung des Schauinsland - Trubelsmattkopf - Knöpflesbrunnenmassivs (westlicher Schwarzwald). - Dipl.-Arb. geol. Inst. Freiburg: 56 S., 4 Kt., 2 Prof., 1 Abb., 1 Diagramm; Freiburg. - [Unveröff.].

Göller, A. (1952): Gletscherspuren im Talgebiet der großen Wiese (südwestlicher Schwarzwald). - Ber. Naturf. Ges. Freiburg i. Br., 42: 45-75, 8 Abb., 1 Kt.; Freiburg.

HANTKE, R. \& RAHM, G. (1976): Das frühe Spätglazial in den Quellästen der Alb (Südlicher Schwarzwald). Vierteljahresschr. Naturf. Ges. Zürich, 121: 293-299, $1 \mathrm{Kt}$.; Zürich.

LIEHL, E. (1982): Landschaftsgeschichte des Feldberggebietes - Altlandschaft - Eiszeit - Verwitterung und Abtragung heute - . - In: Der Feldberg im Schwarzwald. Subalpine Insel im Mittelgebirge. - Die Naturu. Landschaftsschutzgebiete Baden-Württembergs, 12: 13-147, 106 Abb., 6 Tab.; Karlsruhe.
Platz, PH. (1893): Die Glazialerscheinungen des Schwarzwaldes. - Mitt. Großherzogl. Bad. Geol. Landesanst., 2: 837-924, 10 Abb., 2 Taf.; Heidelberg.

Roser, PH. (1899): Zur Kenntnis des Pleistocän im südlichen Schwarzwald. - Diss. Basel: 21 S.; Basel (Birkhäuser).

SCHREPFER, H. (1931): Glazialprobleme im westlichen Hochschwarzwald. - Ber. Naturf. Ges. Freiburg i. Br., 31: 161-210, 1 Kt.; Naumburg.

Steinmann, G. (1896): Die Spuren der letzten Eiszeit im Hohen Schwarzwalde. - Univ.-Festschr. z. Feier d. 70. Geburtstages d. Großherzogs Friedrich: 189-266, 5 Kt., 1 Taf.; Stuttgart.

Manuskript eingegangen am 4. 8. 1986. 


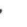

